\title{
Steganographic Algorithm in Spatial Domain
}

Dujan B. Taha

Ghada Mohammad Tahir Qasim

ghada@uomosul.edu.iq

College of Computer Sciences and Mathematics

University of Mosul, Iraq

Received on: 11/10/2006

Accepted on: 24/12/2006

\begin{abstract}
This research deals with design and implementation of a new steganographic algorithm to hide secret messages inside digital images. To achieve security to the transmitted messages, a secret key is used to prevent unauthorized persons from extracting these messages.

Embedding has been achieved using grey and color images as a cover. Matlab is used to implement the algorithm due to the facilities it provides for dealing with digital images and matrices as well as GUI.

Finally, experimental results demonstrate the effieciency of the algorithm in hiding information in imperceptible manner.

Keywords: steganography, message hide digital image.

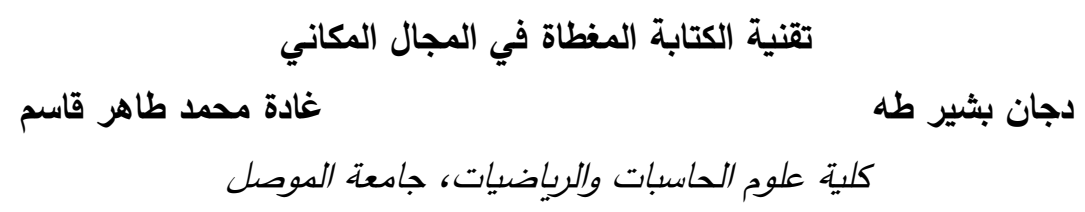

تاريخ قبول البحث: 2006/12/24

تاريخ استلام البحث: 2006/10/11

$$
\begin{aligned}
& \text { الملخص } \\
& \text { تم في هذا البحث تصميم وتتفيذ خوارزمية جديدة لغرض إخفاء الرسائل السرية داخل } \\
& \text { الصور الرقمية ولزيادة سرية الرسائل المنقولة تم استخدام مفتاح سري بإذ لا يمكن استرجاع الرسالة } \\
& \text { دون معرفته أي أن الجهة المخولة التي تعلم المفتاح السري هي التي تستطيع فقط استرجاع الرسالة } \\
& \text { السرية. }
\end{aligned}
$$


تم الإخفاء باستخدام الصور الملونة والرمادية كغطاء كما تم استخدام لغة (Matlab) في هذا البحث وذلك لكون الإخفاء تم في الصور الرقمية التي هي مصفوفات من القيم الرقمية وان لغنة التهاء

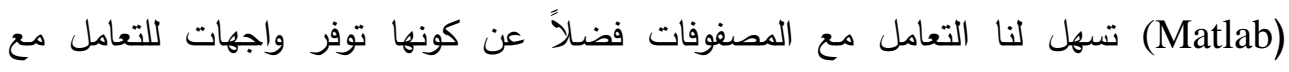

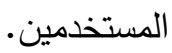

وأخيرا، فان التجارب العملية أثتت كفاءة الخوارزمية المقترحة في إخفاء المعلومات في الصور دون إدراك ذلك. الكلمات المفتاحية: الاخفاء، اخفاء النصوص، الصور الرقمية.

1. مقدمة في إخفاء المعلومات إن فكرة إخفاء المعلومات البسيطة استخدمت منذ مئات السنين،ولكن مع ازدياد استخدام

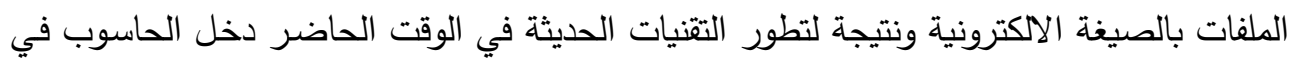

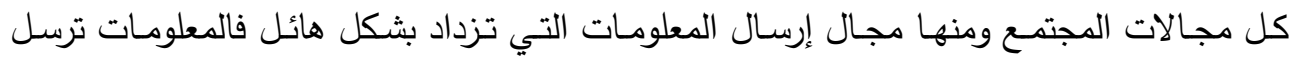

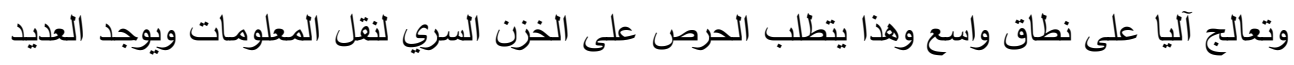

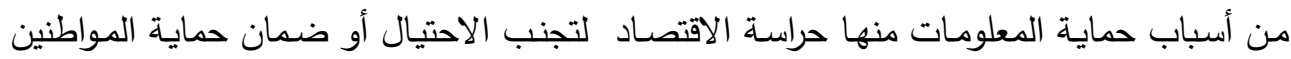

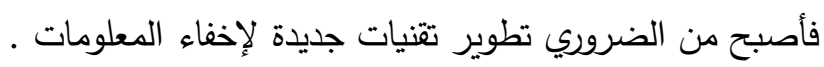

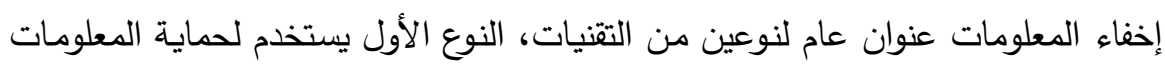

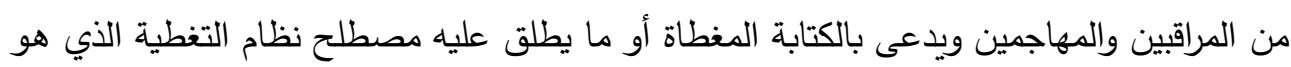

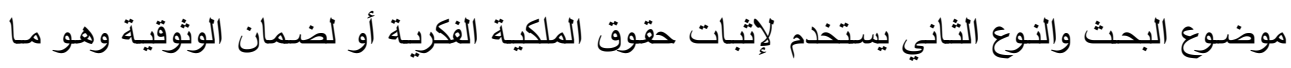
يدعى بالعلامة المائية الرقمية. يوضح الثكل (1)أنواع إخفاء المعلومات وكيف أن إخفاء المعلومات يتفرع إلى مساحات التفاء

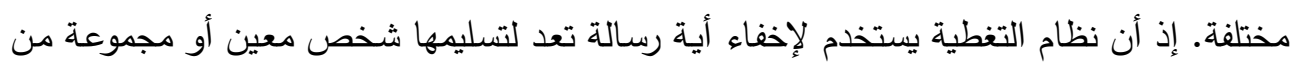

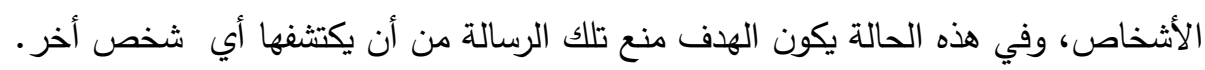

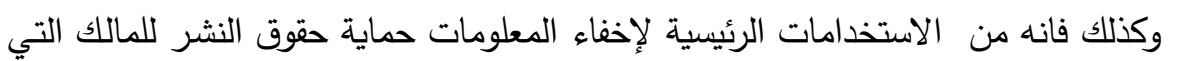

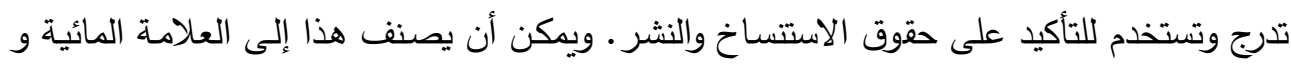

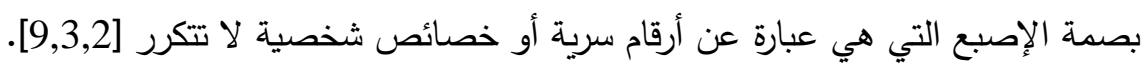


وللمقارنة بين التقنيات المختلفة لتامين سرية الاتصالات نجد أن التشفير يعطينا اتصالا

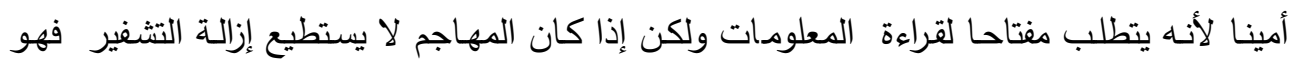
يستطيع بسهولة التحوير في الملف بإذ يجعل الملف غير صـالح للقراءة من قبل المستلم. وعلم الإخفاء يمدنا بأسلوب أمين للاتصالات لا يمكن إزالته دون عمل تغييرات جوهرية على البيانات

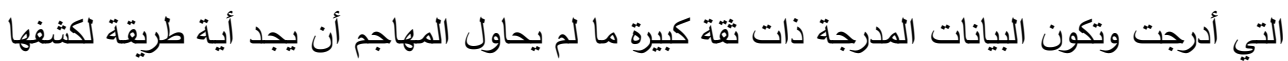

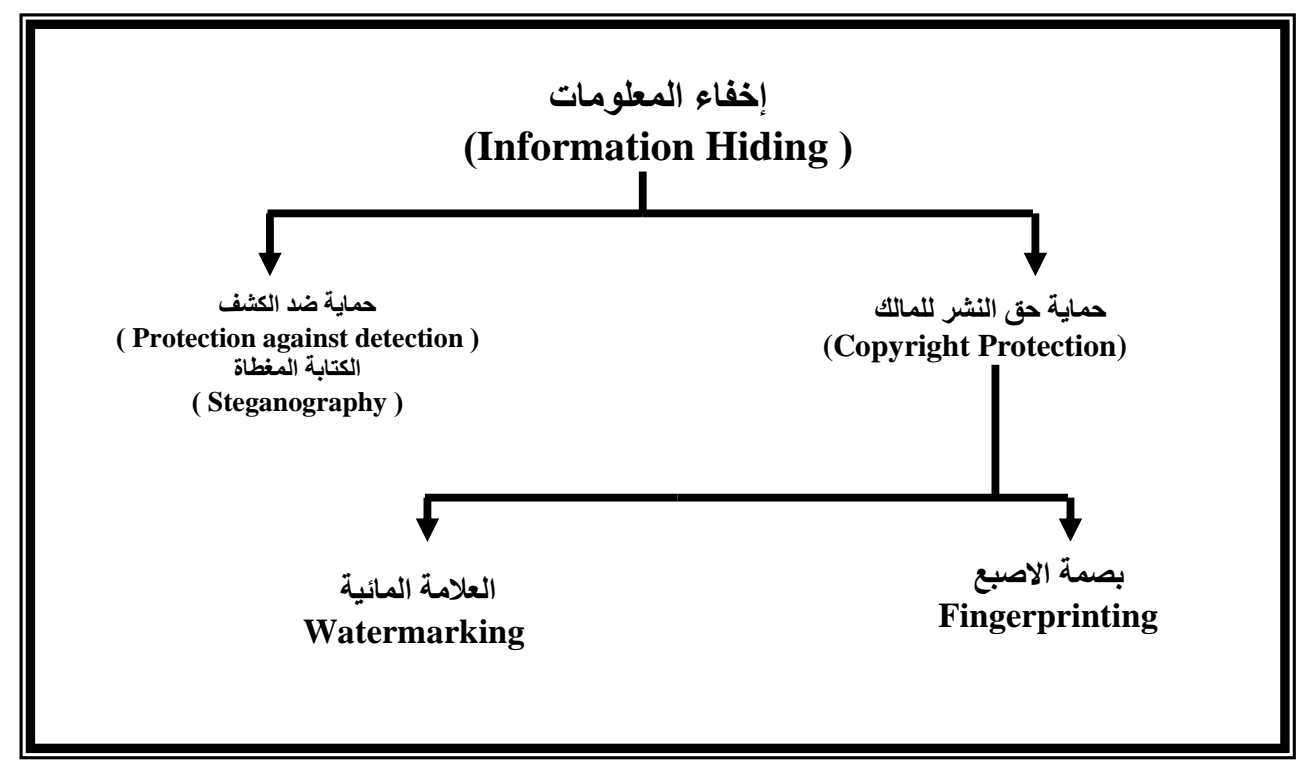

الثكل (1) أنواع إخفاء المعلومات

في تطبيقات إخفاء المعلومات، من غير الممكن أن نحصل على قوة أو متانة للخوارزمية،

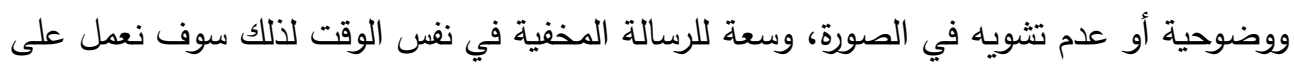

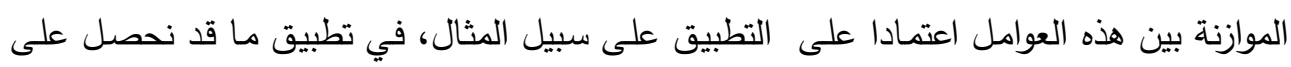
القوة على حساب الوضوحية والسعة [7]. 
وأخيرا فـان التقنيات المستخدمة لإخفـاء المعلومـات تتتوع بالاعتمـاد على كميـة البيانـات المخفية ولكن من الملحوظ عدم توافر طريقة واحدة قادرة على إنجاز جميع الأهداف المطلوبـة من

\section{2. متطلبات إخفاء المعلومات رقميا}

هنـاك عدة تقنيات تضـمين تمكنتـا مسن إخفاء البيانـات في غطـاء معين، وجميع هذه التقنيات يجب أن تحقق عددا من المتطلبات لكي يمكن تطبيق نظريـة إخفاء المعلومـات بصورة صحيحة وفي أدناه مجموعة من المتطلبات الرئيسية [4,1]: 1.الإكمال الصحيح للمعلومات المخفية لدى تضمينها داخل الغطاء الحامل، بإذ أن الرسالة السرية

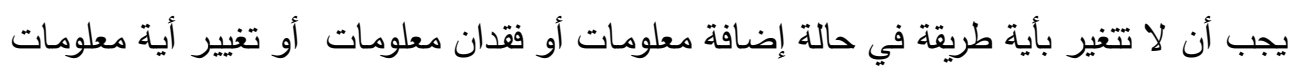
مضمنة بعد إخفائها ويعني تغير البيانات المضمنة فشل العملية .

2.الوسط الناقل الذي يغطي الرسالة السرية يجب أن لا يتغير أيضا وعلى الأقل أن لا تكون تغيراته

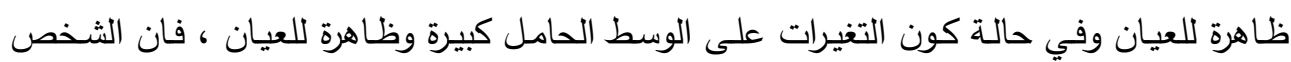
الذي يشاهدها سوف يشك بان هنالك معلومات مخفية داخلها فيحاول أن يفتحها أو يدمرها. 3.في العلامـات المائية يجب أن لا تئثر التغيرات التي تحدث في الرسـالة على متانة العلامـات

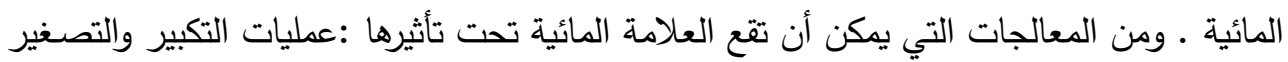

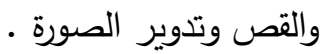
4.يؤخذ بنظر الاعتبار أن المهاجم يثك دائما بوجود معلومات مخفية داخل الغطاء الحامل.

\section{3. نظام التغطية}

نظام التغطية كلمة يونانية الأصل تعني لغويا الكتابة المغطاة إذ أنها تتألف من مقطعين وتعني مغطاة أو سرية و(Graphy) وتعني الكتابة أو الرسم. ويمكن تعريف نظام (Steganos) التغطية على أنه فن وعلم إخفاء المعلومات باستخدام ملف يستخدم كناقل لتلك المعلومات بطريقة لا لاهي تسمح لأي عدو أو مراقب بان يكتشف أن هناك بيانات سرية أخرى مخفية في هذا الملف.والهدف من نظام التغطية إخفاء ماهية الرسالة لمنع أي متطفل خارجي من الثك بوجود رسالة مخفية داخل 
الملف الحامل. إن نظام التغطية يعتبر وسيلة من وسائل الاتصـال السري بأسلوب يخفي وجود

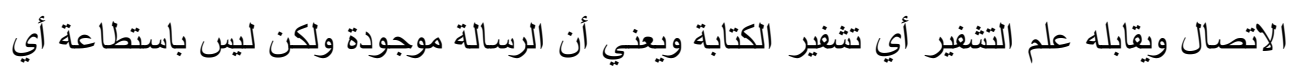

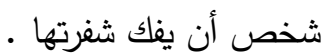

ونظام التغطية ببساطة يأخذ جزءا من المعلومات ويخفيها بملف حاسوبي آخر سواء أكان ذلك الملف صورة أو صوتا أو نصا...الخ يحتوي على مساحات فارغة غير مستخدمة لأن نظام التغطية يستغل المساحات الفارغة وغير المستخدمة ويستبللها بمعلومات أخرى [6,5].

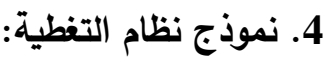
إن النموذج الأساسي لنظام التغطية مبين في الثكل (2). إذ توجد أربعة عناصر في الإخفاء هي [8,6]: * الناقل أو الغطاء (COVER).

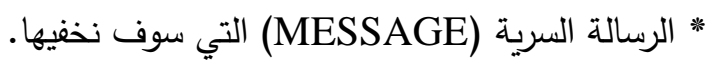

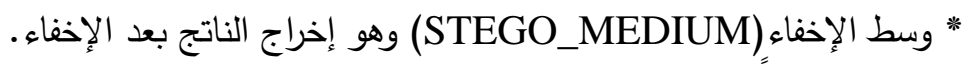
* مفتـاح الإخفاء(Optional_key) يكون اختياريـا ويستخدم لزيـادة درجـة السرية ومـن المدكن تجاوزه. ويمكن تمثيل نظام التغطية باستخدام المعادلة الآتية : 


\section{Cover_medium+Embedded Message+Stego_key=Stego_medium}

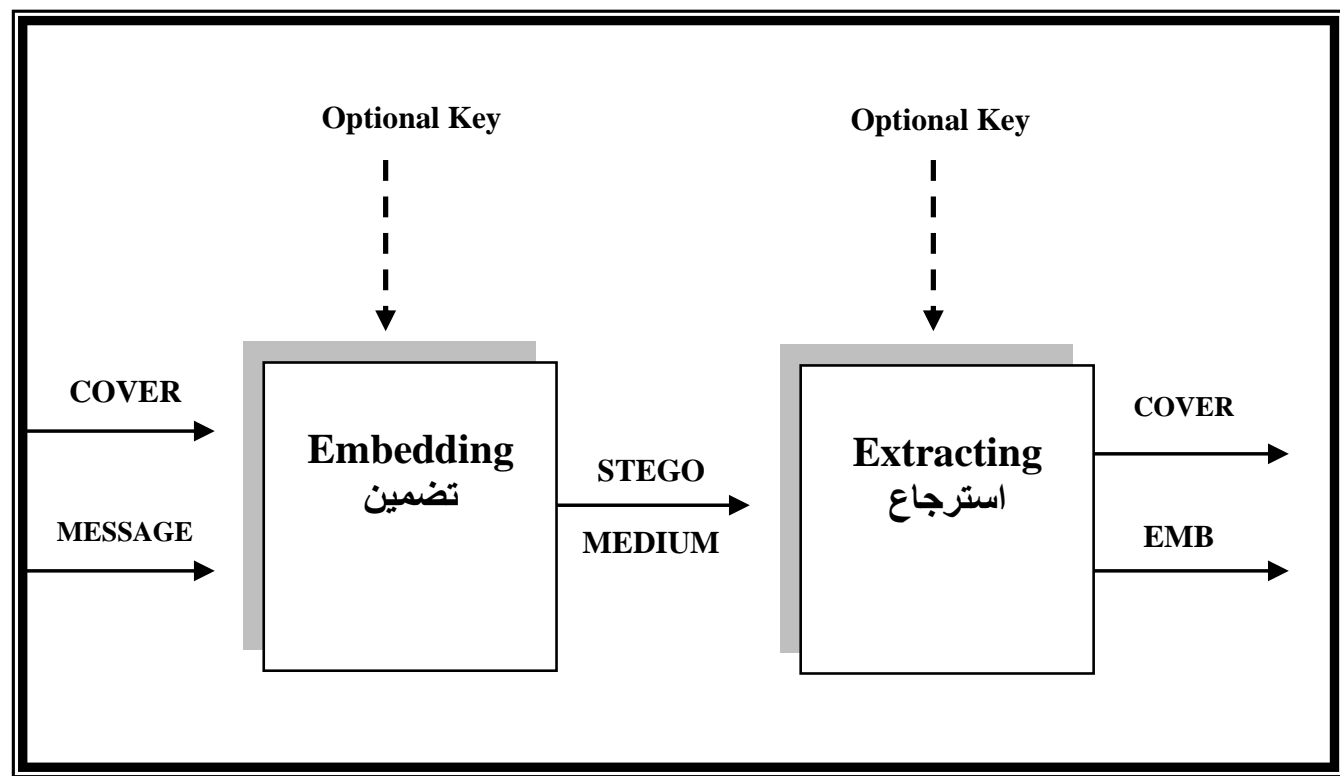

الثكل (2) النموذج الأساسي لنظام التغطية

5. 5 5 تصميم الخوارزمية المقترحة:

$$
\text { 1.مرحلة توليد الرسالة: }
$$

تبدأ هذه المرحلة بقراءة الرسالة المراد إخفاؤها داخل صورة ثم يتم تحويل أحرف الرسالة

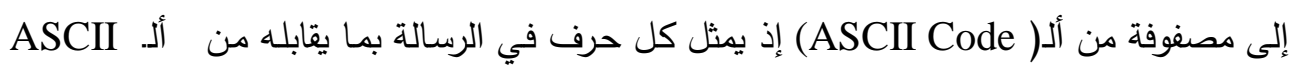

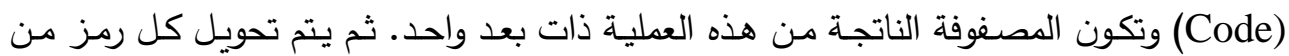

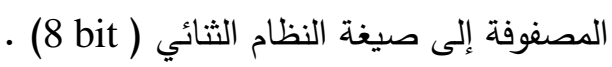

ومن ثم يتم دمج هذه القيم الثنائية في مصفوفة ذات بعد واحد ولزيـادة سرية الرسالة

المضمنة نقوم بإبدال مواقع قيم المصفوفة باستخدام مواقع عشوائية يتم توليدها من دالة دالة الأرقام

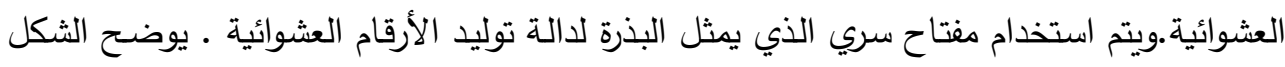

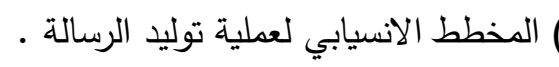




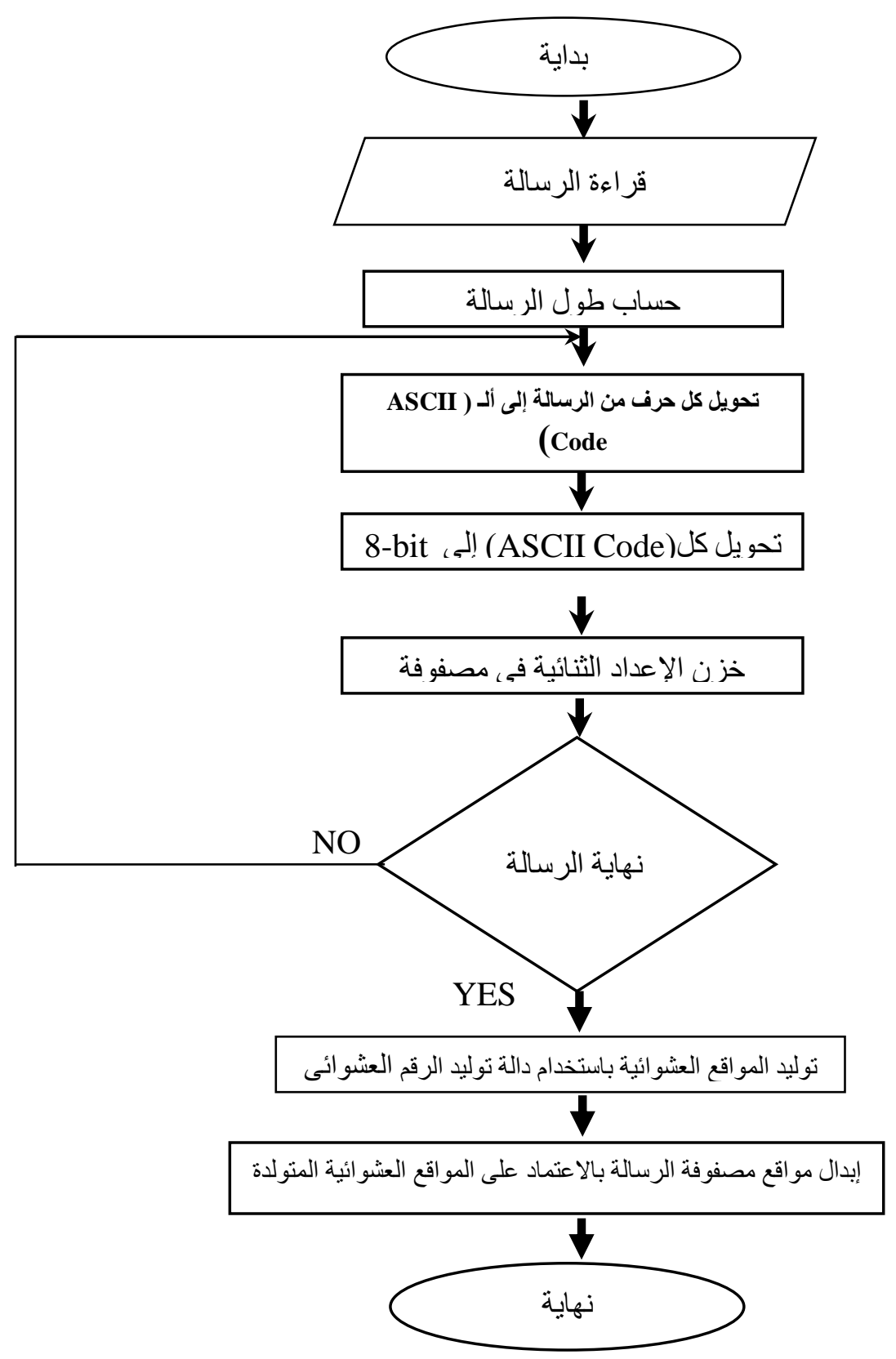

الثكل (3 ) المخطط الانسيابي لعملية توليد الرسالة 
2. مرحلة تضمين الرسالة: يوضح الثكل (4) عملية تضمين الرسالة إذ يكون الإدخال هنا هو الرسالة (M) والصورة الأصلية (P) ويكون الإخراج لهذه المرحلة هو الصورة بعد تضمين الرسالة فيها (P).

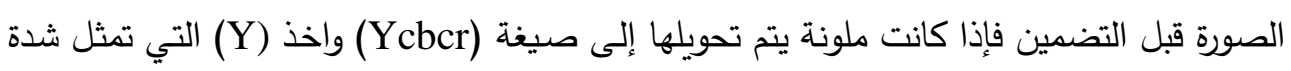
الإضاءة لغرض التضمين إنول تتلخص خوارزمية تضمين الرسالة بالخطوات الآتية :

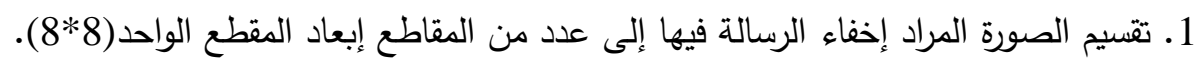
2. إيجاد عدة حسابات للمقطع الواحد تتمثل بما يأتي : إعلئ

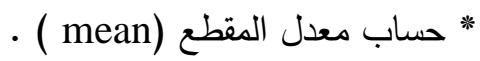

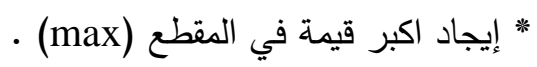
* إيجاد اقل قيمة في المقطع (min). * إيجاد القيم التي هي أعلى من المعدل (hi) وحساب معدل هذه القيم (mhi) * إيجاد القيم التي هي اقل من المعدل (low) وحساب معدل هذه القيم (mlow).

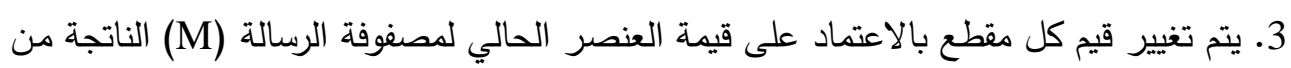

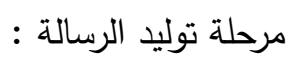
a إذا كان العنصر الحالي لمصفوفة الرسالة هو (1) فيتم تغيير قيم عناصر المقطع كالآتي:

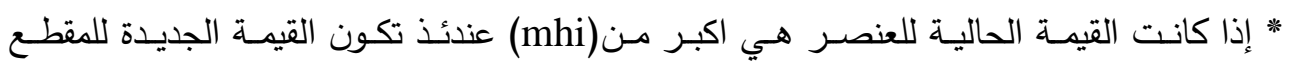
هي). * أما إذا كانت القيمة الحالية للعنصر هي اكبر أو تساوي (mlow) و اقل من أل (mean) فتكون القيمة الجديدة للمقطع هي (mean). * أمـا إذا لم تحقق أياً من الشروط أعلاه عندئذ تكون القيمة الجديدة للمقطع هي القيمة الحالية للمقطع مضافا إليها قيمة ثابتة (م) وهذه القيمة تم تحديدها في البرنامج بحيث لا تؤثر في الصورة المضمنة .

b أما في حالة كون العنصر الحالي لمصفوفة الرسالة هو (0) فيتم تغيير قيم المقطع كالأتي:

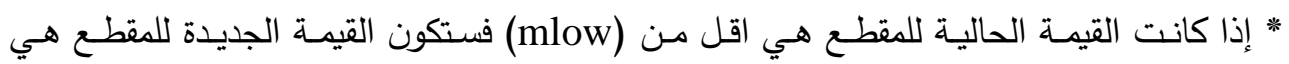
- (min) 
* إمـا إذا كانت القيمـة الحاليـة للمقطع هي اكبر أو تسـاوي (mean) واقل من(mhi) فعندها ستكون القيمة الجديدة للمقطع هي (mean).

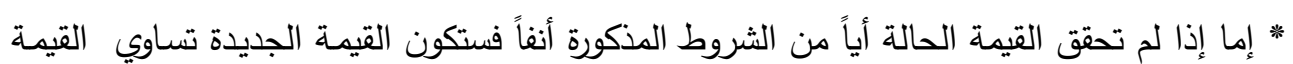

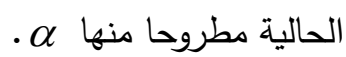

وتستمر عملية التضمين هذه إلى نهاية عناصر مصفوفة الرسالة (M) , ويمثل الثكل (5) الخخط

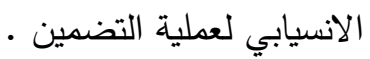

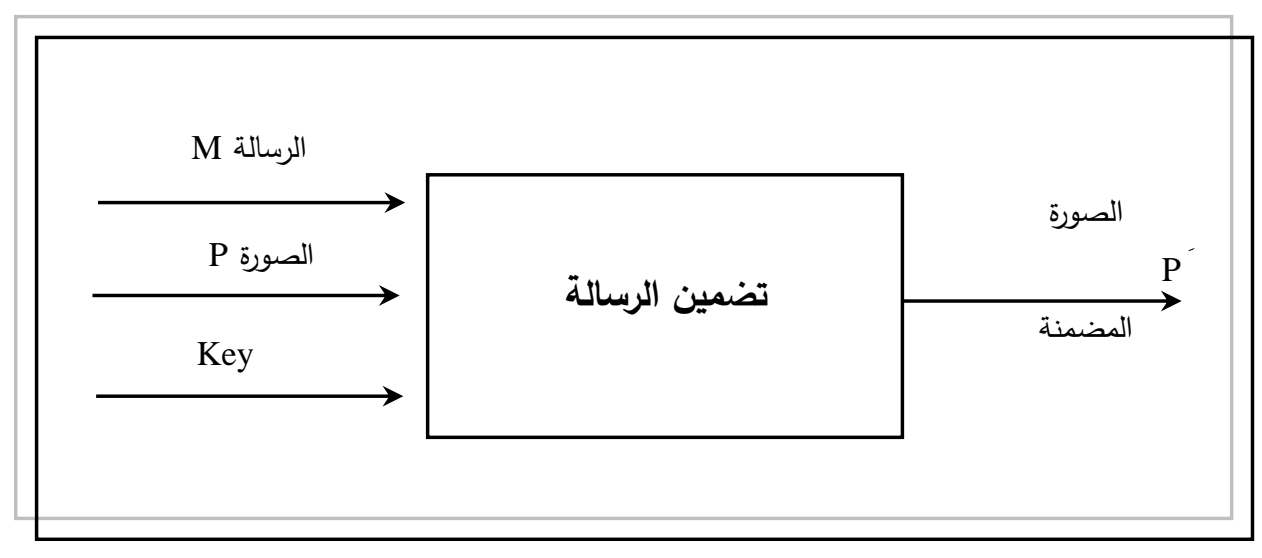

الثكل(4) تضمين الرساية

3. استرجاع الرسالة المضمنة

إن عملية استرجاع الرسالة من الصورة هي العملية المعاكسة لعملية التضمين ,ويمثل

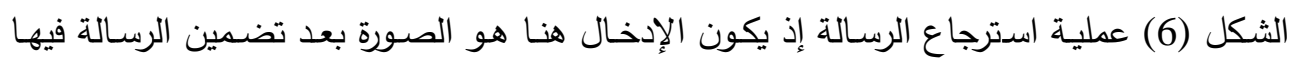

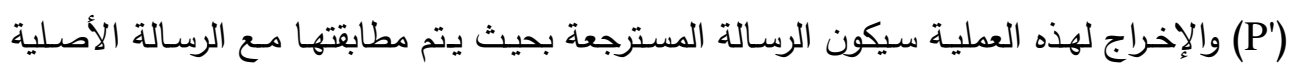

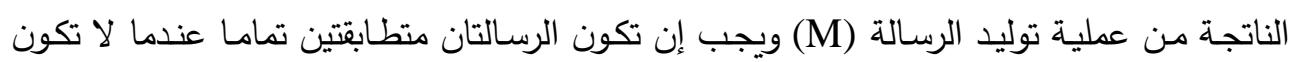

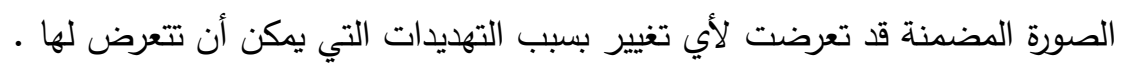




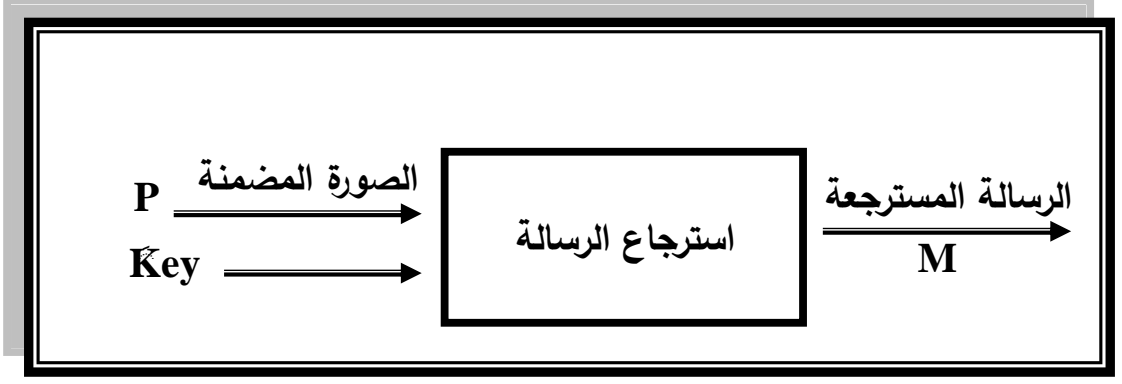

\section{الثكل (6) استرجاع الرسالة}

إن عملية استرجاع الرسالة تتلخص بمرحلتين هما الاسترجاع وعرض الرسالة. تتلخص خوارزمية الاسترجاع بما يأتي:

يتم تقسيم الصورة الأصلية إلى عدد من المقاطع المشابهة للمقاطع في عملية التضمين وكذلك يتم تقسيم الصورة الناتجة من عملية تضمين الرسالة إلى نفس العدد من المقاطع رومن ثم يتم

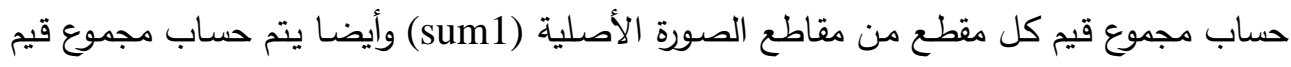
كل مقطع من مقاطع الصـورة بعد التضـمين (sum2) وبعدها يتم فحص مص إذا كـان sum1 لأحد المقاطع في الصورة الأصلية اكبر من sum2 للمقطع المقابل له في الصورة المضمنة فعندها يكون البت المسترجع هو صفر إذا كان sum1 اقل أو يساوي sum2 فعندها يكون البت المسترجع هو

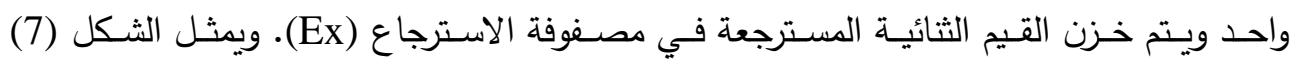
المخطط الانسيابي لعملية الاسترجاع. 


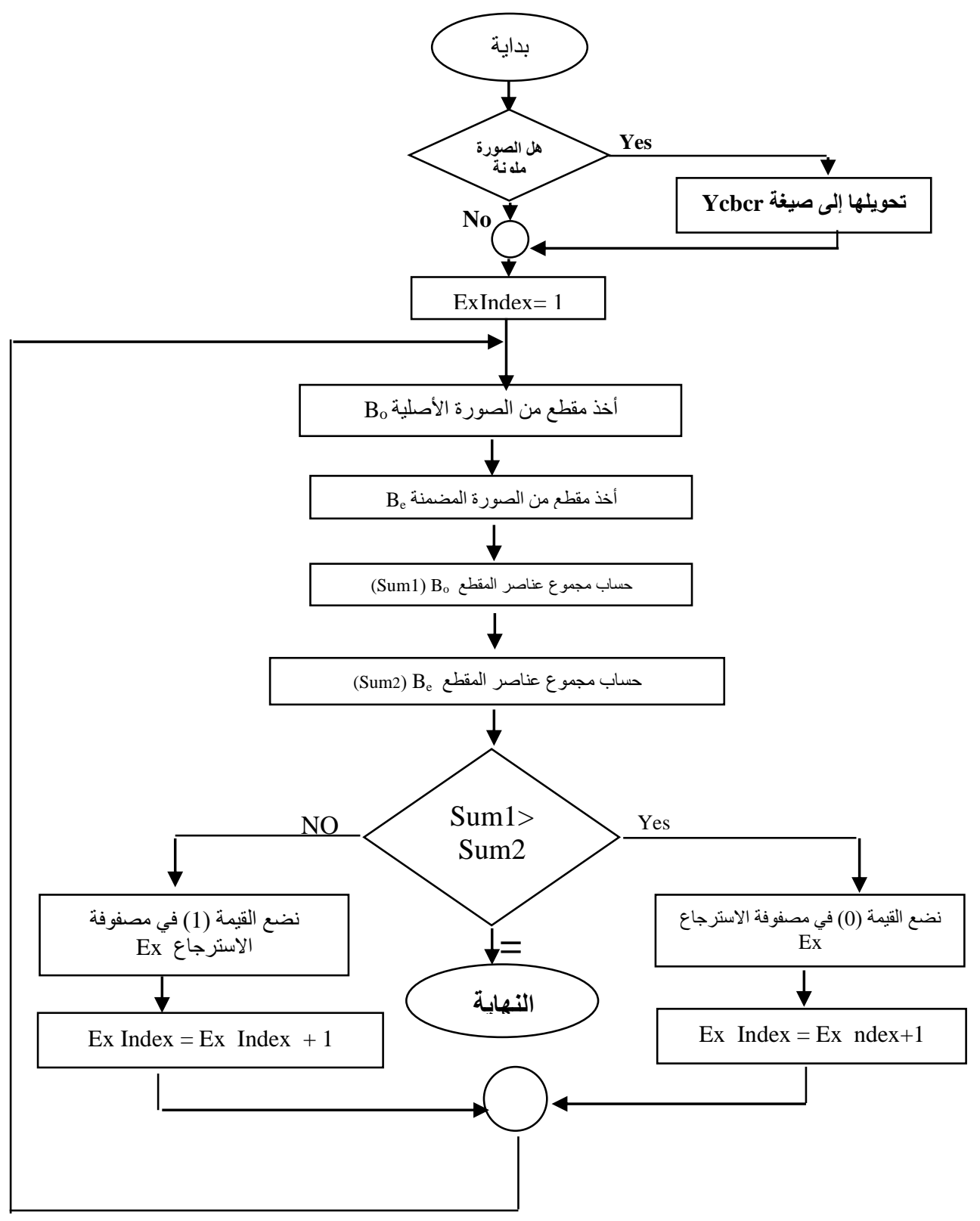

الشكل (7) المخطط الآسيابي لعملية استرجاع الرسالة 
إن مرحلة عرض الرسالة هي العملية المعاكسة لعملية توليد الرسالة إذ يكون الإدخال هنا هو مصفوفة الاسترجاع التي تحوي على القيم الثنائية المسترجعة من الصورة المضمنة، وبما أنه تم

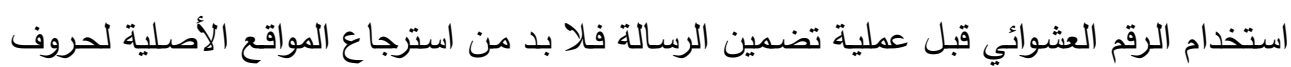
الرسالة المسترجعة قبل عرضها فيتم استخدام نفس المفتاح لتوليد نفس السلسة العشوائية قبل تحويل

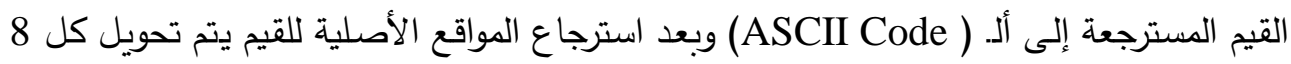

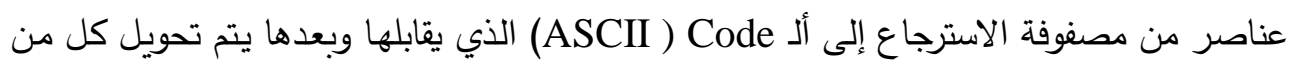
هذه الرموز إلى الحرف المقابل له وأخيرا توضع جميع الحروف في مصفوفة لغرض عرضئ الهنها. ويمثل الشكل (8) المخطط الانسيابي لعملية عرض الرسالة.

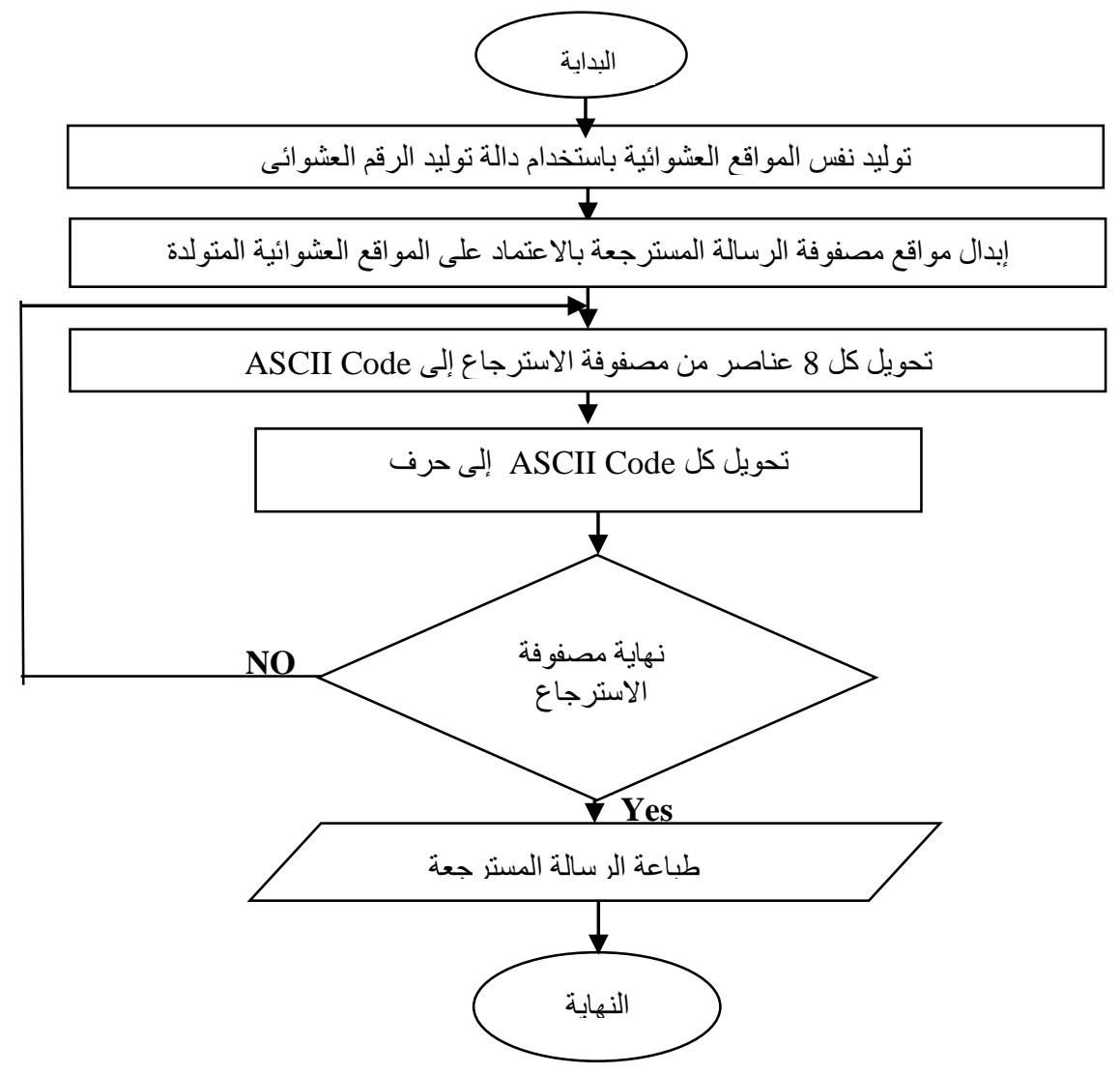

الثكل (8) المخطط الانسيابي لعملية عرض الرسالة 
6. - - النتائج والاستنتاجات

1

- التضمين

إن واجهة التضمين ( الثكل 9) تتألف من مجموعة مكونات هي:-

Enter message -a

يتم إدخال الرسالة المراد إخفاؤها.

Enter Key -b

يتم إدخال المفتاح السري للرسالة ويمكن تغيير المفتاح في أي وقت عند الحاجة.

Choose the Cover $-\mathrm{c}$

يتم اختيار صورة من بين مجموعة من الصور .

Next $-d$

يتم الانتقال إلى برنامج التضمين وعرض النتيجة وهي الصورة بعد تضمين الرسالة فيها.

إذ عند اختيار صورة ملونة تكون النتيجة كما موضحة في الثكل (10) وإذا تم اختيار صور رمادية تكون النتيجة كما في الثكل (11). تم حساب PSNR والتي تقاس بوحدة dB لـعرفة فيما إذا كان هناك تشوه في الصورة ولوحظ أن الصورة الناتجة خالية من أي تشوه إذ كانت قيمة PSNR تساوي 45.59dB و 41.99dB للصورة الملونة والرمادية على الترتيب وهي قيمة جيدة جدا.

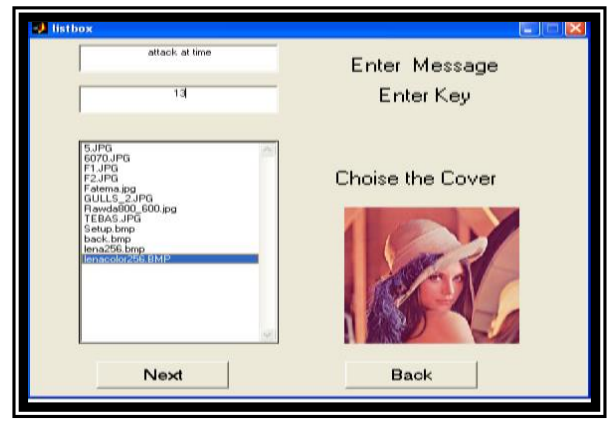

الشكل (9) واجهة التضمين 


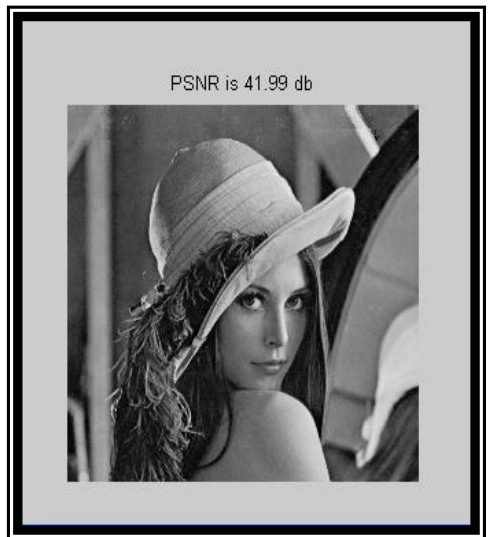

الثكل (11)(التضمين في الصورة الرمادية

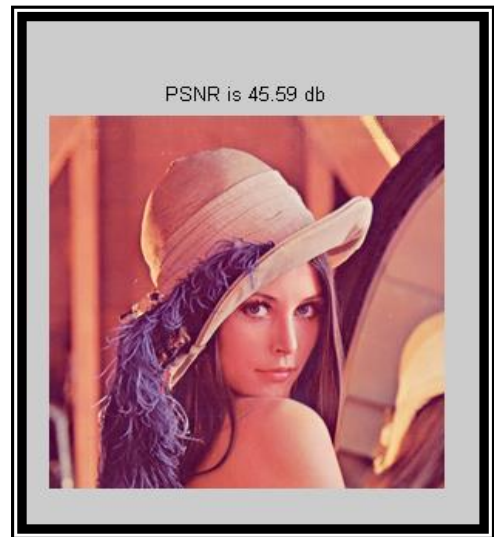

الثكل (10)التضمين في الصورة الملونة

- الاسترجاع -

إن واجهة الاسترجاع (الثكل 12) تتألف من المكونات الأتية:

Enter key -a

يتم إدخال نفس المفتاح السري المستخدم في تضمين الرسالة لغرض استرجاعها.

Next $-b$

يتم الانتقال إلى برنامج الاسترجاع وعرض الرسالة المسترجعة من الصورة كما في الثكل (13).

Back -c

يتم الرجوع إلى الواجهة الرئيسية .

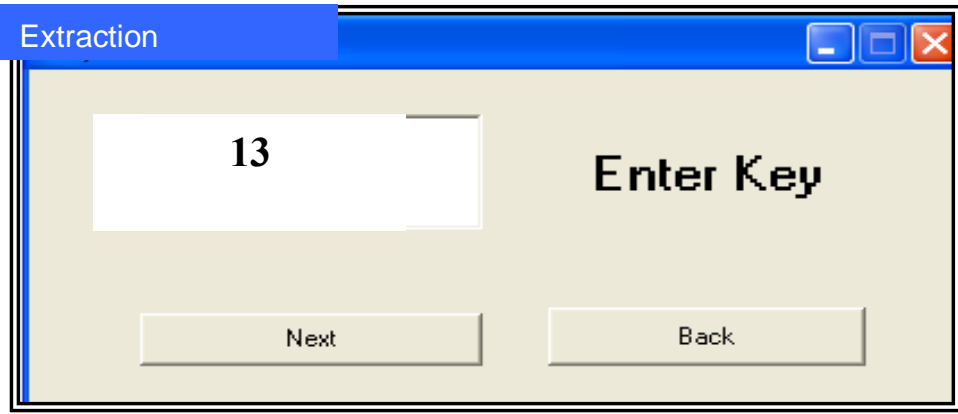

الثكل (12) واجهة الاسترجاع 


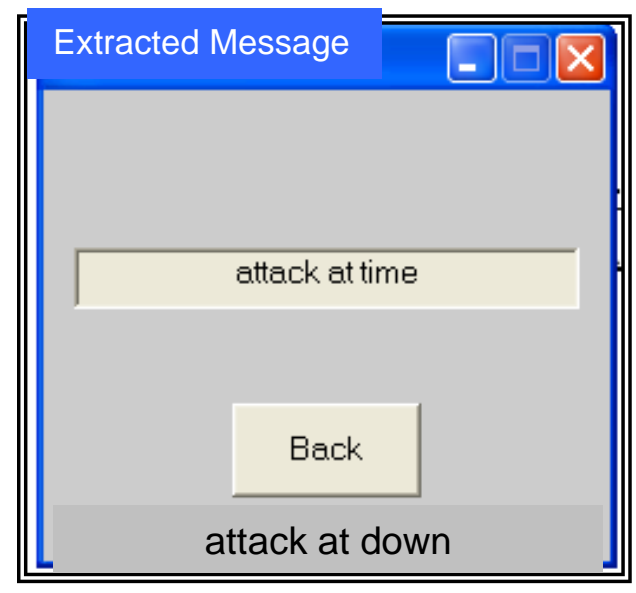

الثكل (13) واجهة عرض الرسالة المسترجعة

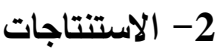

1. يوفر البرنامج واجهة مرئية سهلة الاستخدام من قبل المستخدم ومرنة وواضحة تمكن المستخدم من تحديد العملية واختيار الصورة.

2. الخوارزمية المستخدمة في هذا المشروع توفر سرية كبيرة في إرسال الرسائل والتي تتضمن ما يأتي: أ. استخدام الإبـدال العشـوائي لمواقـع المراتب الثنائيـة للرسـالة الأصـلية وهذا يـوفر سـرية للرسـالة المرسلة.

ب. القيام بعملية الإبدال العشوائي عن طريق استخدام مفتاح سري خاص لا يمكن إرجاع الرسالة الأصلية من دون معرفته ويكون معروف من قبل الثخص المخول فقط.

3. تم تتفيذ البرنامج على الصور الملونة والرمادية إذ أن الصور الملونة قد يكون التشوه فيها واضحا

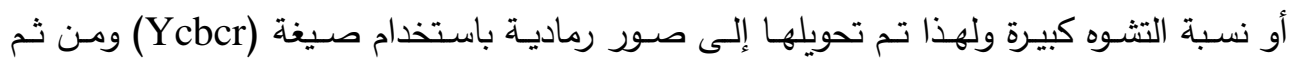

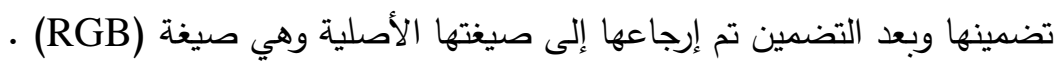

4. يمكن تتفيذ البرنامج على صور غير محددة الأبعاد واكبر من حجم الرسالة المراد إخفاؤها. 5. تم الحصول على نتائج جيدة في الإخفاء باستخدام هذه الخوارزمية إذ تم حساب نسبة التثوه في الصور الناتجة بعد التضمين ولوحظ إنها نسبة جيدة جدا تثير إلى عدم حصول أي تثوه ملحوظ. 


\section{المصادر}

[1] Bender, W. ; D. Gruhl, ; N. Morimoto, and A. Lu (1996) "Techniques for data hiding", IBM Systems Journal, Vol. 35.

[2] Bret, D. (2002) "A detailed look steganographic techniques and their use in open-system environment" ,SANS Institute.

[3] Cummins, J.; P. Diskin, and R. Parlett (2000) "Steganography and digital watermarking", school of computer science, University of Bermingham.

[4] Hopper, N.; J. Langford, and L. Ahn (2002) “ Provably secure steganography", CRYPTO August 2002.

[5] Johnson, N. and Z. Duric, (2000) Information hiding: steganography and watermarking attacks and countermeasures, Kluwer Academic Publishers.

[6] Karzenbeisser, S. and F. Petitcolas (2000) Information hiding techniques for steganography and digital watermarking, Artech House.

[7] Kharrazi, M. and H. Sencar, (2004) "Image steganography: concepts and practice".

[8] Law, C. (1995) "Steganography hiding writing", Information Network.

[9] Petitcolas, F. and R. Anderson (1999) "Information hiding-A survey", IEEE. 